Article

\title{
On the Kernel of a Polynomial of Scalar Derivations
}

\section{Savin Treanţă}

Department of Applied Mathematics, University Politehnica of Bucharest, 060042 Bucharest, Romania; savin.treanta@upb.ro

Received: 6 March 2020; Accepted: 20 March 2020; Published: 2 April 2020

Abstract: In this paper, by using a vector variable, the procedure of characteristic systems allows us to describe the kernel of a polynomial of scalar derivations by solving Cauchy Problems for the corresponding system of ODEs. Moreover, a gradient representation for the associated Cauchy Problem solution is derived.

Keywords: scalar derivation; characteristic system; Lie algebra; gradient system; kernel

MSC: 34A26; 15A03; 35C99

\section{Introduction and Problem Formulation}

The gradient-type representations for some solutions, Lie algebras, gradient systems in a Lie algebra, algebraic representation of gradient systems and their integral manifolds, have been studied for a long time, with remarkable results, by Vârsan [1] and Barbu et al. [2]. Apart from the linear higher order PDEs, the characteristic system method is intensively used for solving linear or nonlinear SPDEs and in this respect, we mention Iftimie et al. [3]. For other different but related viewpoints on this subject, the reader is directed to Friedman [4], Sussmann [5], Crandall and Souganidis [6], Sontag [7], Bressan and Shen [8], Evans [9], Brezis [10], Parveen and Akram [11], Treanţă and Vârsan [12], Treanţă [13].

In this paper, by using a vector variable, the procedure of characteristic systems allows us to describe the kernel of a polynomial of scalar derivations by solving Cauchy Problems for the corresponding system of ODEs. Moreover, a gradient representation for the associated Cauchy Problem solution is derived. As the main motivation of this study, the mathematical framework developed in this work can be extended for the study of some higher-order hyperbolic, parabolic or Hamilton-Jacobi equations involving a finite set of derivations. For instance, a simple $m$-th order Hamilton-Jacobi equation has the following expression

$$
(\vec{Z})^{m}(\varphi)(t, x)=\sum_{k=0}^{m-1} a_{k}(t)(\vec{Z})^{k}(\varphi)(t, x)+f(t),(t, x) \in[0, T] \times R^{n},
$$

where $\vec{Z}: C^{1}\left([0, T] \times R^{n} ; R\right) \rightarrow C\left([0, T] \times R^{n} ; R\right)$ is a linear application defined by

$$
\vec{Z}(\varphi)(t, x)=\partial_{t} \varphi(t, x)+\left\langle\partial_{x} \varphi(t, x), X(t, x)\right\rangle, t \in[0, T], x \in R^{n}
$$

(see $\vec{Z}$ as being generated by the vector field $Z(z)=\operatorname{col}(1, X(z)), \quad z=(t, x)$, where $X \in$ $\left(C_{b}^{1} \cap C^{m}\right)\left([0, T] \times R^{n} ; R^{n}\right), m \geq 2$, and the index $b$ of $C_{b}^{1}$ is for bounded $)$ and $\left\{a_{k}, f: 0 \leq k \leq\right.$ $m-1\} \subseteq C([0, T] ; R)$. Using standard notation, $\varphi=y_{0},(\vec{Z})^{k}(\varphi)=y_{k}, 0 \leq k \leq m-1$, rewrite (1) as a system of Hamilton-Jacobi equations

$$
\vec{Z}\left(y_{0}\right)(t, x)=y_{1}(t, x), \quad \cdots \quad, \vec{Z}\left(y_{m-2}\right)(t, x)=y_{m-1}(t, x),
$$




$$
\vec{Z}\left(y_{m-1}\right)(t, x)=\sum_{k=0}^{m-1} a_{k}(t) y_{k}(t, x)+f(t),(t, x) \in[0, T] \times R^{n} .
$$

A classical solution $y(t, x)=\left(y_{0}(t, x), \ldots, y_{m-1}(t, x)\right) \in R^{m},(t, x) \in[0, T] \times R^{n}$, associated with (2), means a first order continuously differentiable mapping $y \in C^{1}\left([0, T] \times R^{n} ; R^{m}\right)$ satisfying (2) for any $(t, x) \in[0, T] \times R^{n}$. The first component of a solution verifying (2) stands for a classical solution of the higher order Hamilton-Jacobi equation (1). It is well known that the characteristic system method is associated with the classical solutions of the PDEs (at least continuous functions). In the previous context, a solution of the corresponding higher order PDEs involves a characteristic system containing a bounded variation component as solution for some ODEs.

Throughout this paper, let $0 \in I \subseteq R$ be an open interval. Consider a polynomial of the scalar derivation $\frac{d}{d t}$,

$$
P_{m}\left(t ; \frac{d}{d t}\right)=a_{1}(t)+a_{2}(t)\left(\frac{d}{d t}\right)+\cdots+a_{m}(t)\left(\frac{d}{d t}\right)^{m-1}-\left(\frac{d}{d t}\right)^{m},
$$

where $m \geq 1, a_{j} \in L_{\infty}(I), j \in\{1,2, \ldots, m\}$. Define

$$
H_{\infty}^{m}(I)=\left\{h \in C^{m-1}(I):\left(\frac{d}{d t}\right)^{m}(h) \in L_{\infty}(I)\right\}
$$

and consider $\operatorname{Ker}\left(P_{m}\right) \subseteq H_{\infty}^{m}(I)$, where

$$
\operatorname{Ker}\left(P_{m}\right)=\left\{h \in H_{\infty}^{m}(I): P_{m}\left(t ; \frac{d}{d t}\right)(h)(t)=0 \text {, a.e. } t \in I\right\} .
$$

The procedure of characteristic systems (see Friedman [4], Vârsan [1]) allows us to describe $\operatorname{Ker}\left(P_{m}\right)$ by solving Cauchy Problems for the corresponding system of ODEs using a vector variable

$$
y=\operatorname{col}\left(y_{1}, y_{2}, \ldots, y_{m}\right), \frac{d y}{d t}=A y+\sum_{i=1}^{m} a_{i}(t) B_{i} y, y(0)=y_{0} \in R^{m}
$$

Here, the $(m \times m)$ constant matrices $A$ and $B_{i}, i=\overline{1, m}$, are defined by

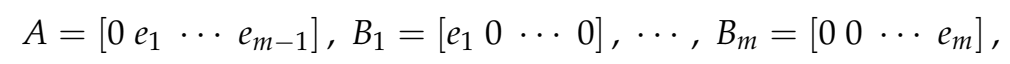

where $\left\{e_{1}, \ldots, e_{m}\right\}$ is the canonical basis and $0 \in R^{m}$ is the origin. By definition

$$
\left[B_{i}, B_{j}\right]:=B_{j} B_{i}-B_{i} B_{j}, i, j \in\{1,2, \ldots, m\}, \quad \text { (Lie bracket), }
$$

and making a direct computation, we get

$$
O=\left[B_{i}, B_{j}\right], i, j \in\{1,2, \ldots, m\},
$$

with $O$ - null matrix, and

$$
A^{m}=O, \quad(A \text { is a nilpotent matrix }) .
$$

The Cauchy Problem solution for (4) is represented by

$$
y\left(t ; y_{0}\right)=[\exp A t] \hat{y}\left(t ; y_{0}\right), t \in I,
$$


where $\left\{\hat{y}\left(t ; y_{0}\right): t \in I\right\}$ fulfils the following linear system (initial value problem)

$$
\frac{d y}{d t}=\sum_{i=1}^{m} a_{i}(t) A_{i}(t) y, t \in I, y(0)=y_{0} \in R^{m}
$$

By using the linear mapping $\operatorname{ad}_{A}: M_{m \times m} \rightarrow M_{m \times m}, \operatorname{ad}_{A}(B):=B A-A B$ (see $[A, B]$ ), write the $(m \times m)$ matrices

$$
A_{i}(t):=[\exp (-t A)] B_{i}[\exp t A], i \in\{1,2, \ldots, m\},
$$

as follows

$$
A_{i}(t)=B_{i}+\frac{t}{1 !} \operatorname{ad}_{A}\left(B_{i}\right)+\cdots+\frac{t^{m-1}}{(m-1) !} \operatorname{ad}_{A}^{m-1}\left(B_{i}\right), i \in\{1, \ldots, m\} .
$$

In addition, taking into account (9), (10) and (14), we get

$$
A_{i}(t)=\left[\exp t \operatorname{ad}_{A}\right]\left(B_{i}\right), i \in\{1,2, \ldots, m\}, t \in I .
$$

Denote $N=m^{2}$ and define $N$ matrices $\left\{C_{1}, C_{2}, \ldots, C_{N}\right\} \subseteq M_{m \times m}$, as follows

$$
\begin{gathered}
\left\{C_{1}, C_{2}, \ldots, C_{N}\right\}=\left\{\operatorname{ad}_{A}^{k}\left(B_{1}\right): k \in\{0,1,2, \ldots, m-1\}\right\} \cup \cdots \cup \\
\left\{\operatorname{ad}_{A}^{k}\left(B_{m}\right): k \in\{0,1,2, \ldots, m-1\}\right\} .
\end{gathered}
$$

Moreover, let $\left\{\alpha_{1}(t), \alpha_{2}(t), \ldots, \alpha_{N}(t): t \in I\right\}$ be given by

$$
\begin{gathered}
\left\{\alpha_{1}(t), \ldots, \alpha_{m}(t)\right\}=a_{1}(t)\left[1, \frac{t}{1 !}, \ldots, \frac{t^{m-1}}{(m-1) !}\right], \\
\vdots \\
\left\{\alpha_{N-m+1}(t), \ldots, \alpha_{N}(t)\right\}=a_{m}(t)\left[1, \frac{t}{1 !}, \ldots, \frac{t^{m-1}}{(m-1) !}\right] .
\end{gathered}
$$

With these notations, we write ODE (12) as follows

$$
\frac{d y}{d t}=\sum_{j=1}^{N} \alpha_{j}(t) Y_{j}(y), t \in I, y(0)=y_{0}
$$

where $Y_{j}(y):=C_{j} y, j \in\{1,2, \ldots, N\}$.

\section{Main Results}

In this section, the main results of the present paper are formulated and proved.

Theorem 1. Consider $\left\{C_{1}, C_{2}, \ldots, C_{N}\right\}$ defined in (16), with $N=m^{2}$. Then, $\left\{C_{1}, C_{2}, \ldots, C_{N}\right\}$ is a basis for $M_{m \times m}$ and

$$
\left\{Y_{1}(y)=C_{1} y, Y_{2}(y)=C_{2} y, \ldots, Y_{N}(y)=C_{N} y\right\}
$$

is a system of generators for the Lie algebra $L\left(Y_{1}, \ldots, Y_{N}\right) \subseteq C^{\infty}\left(R^{m} ; R^{m}\right)$ generated by $\left\{Y_{1}, \ldots, Y_{N}\right\}$.

Proof. By direct computation, we rewrite the matrices $\left\{C_{1}, C_{2}, \ldots, C_{N}\right\}$ as follows:

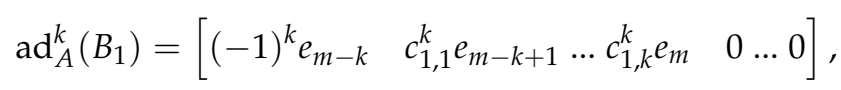


for some constants $c_{1, j}^{k}, 0 \leq k \leq m-1$. For any $1 \leq i \leq m$, we get

$$
\operatorname{ad}_{A}^{k}\left(B_{i}\right)=[\underbrace{0 \ldots 0}_{(i-1) \text { times }}(-1)^{k} e_{m-k} \quad c_{i, 1}^{k} e_{m-k+1} \ldots c_{i, k(i)}^{k} e_{m-k+k(i)} \quad 0 \ldots 0],
$$

where $k(i):=\min \{k, m-i\}$ and $c_{i, j}^{k}$ are some constants.

The particular structure given in (20) leads us directly to the conclusion that $\left\{C_{1}, C_{2}, \ldots, C_{N}\right\} \subseteq$ $M_{m \times m}$ are linearly independent. Therefore, $\left\{C_{1}, C_{2}, \ldots, C_{N}\right\}$ is a basis for $L\left(C_{1}, C_{2}, \ldots, C_{N}\right)=M_{m \times m}$ and using

$$
L\left(Y_{1}, Y_{2}, \ldots, Y_{N}\right)=\left\{C y: C \in L\left(C_{1}, C_{2}, \ldots, C_{N}\right)\right\}
$$

we get that $\left\{Y_{1}, Y_{2}, \ldots, Y_{N}\right\}$ is a system of generators for $L\left(Y_{1}, Y_{2}, \ldots, Y_{N}\right)$. The proof is complete.

The next remark contains several mathematical tools (some of these, introduced in Vârsan [1]) and their hints, which are necessary for proving Theorem 2.

Remark 1. Consider the linear vector fields $Y_{j}(y):=C_{j} y, j \in\{1, \ldots, N\}, y \in R^{m}$, where $\left\{C_{1}, C_{2}, \ldots, C_{N}\right\} \subseteq$ $M_{m \times m}$ is a basis (see Theorem 1). Let $L\left(Y_{1}, \ldots, Y_{N}\right)$ be the finite dimensional Lie algebra generated by $\left\{Y_{1}, \ldots, Y_{N}\right\}$. Then, the following statements are valid:

$$
\operatorname{dim} L\left(Y_{1}, \ldots, Y_{N}\right)\left(y_{0}\right)=\operatorname{dim}\left[\operatorname{span}\left\{Y_{1}\left(y_{0}\right), \ldots, Y_{N}\left(y_{0}\right)\right\}\right]=m,
$$

for any $y_{0} \neq 0 \in R^{m}$;

$$
y(p ; \lambda)=G(p) \lambda:=\left[\exp t_{1} C_{1}\right] \cdots\left[\exp t_{N} C_{N}\right] \lambda,
$$

with $p=\left(t_{1}, \ldots, t_{N}\right) \in R^{N}, \lambda \in R^{m}$, satisfies a gradient system (GS) in $L\left(Y_{1}, Y_{2}, \ldots, Y_{N}\right)$ (see Vârsan [1] for more details; $G(p) \in M_{m \times m}, p \in R^{N}$, is solution for a (GS) in $M_{m \times m}$ )

$$
\partial_{p} y(p ; \lambda)=\left\{Y_{1}, \ldots, Y_{N}\right\}(y(p ; \lambda)) A(p), p \in R^{N},
$$

where $A(p)$ is an $(N \times N)$ analytic matrix fulfilling $A(0)=I_{N}$ and det $A(p) \neq 0$ for $p \in B(0, \rho) \subseteq R^{N}$; if

$$
\varphi\left(y\left(p ; y_{0}\right)\right)=\text { const. }\left(=\varphi\left(y_{0}\right)\right), \forall p \in B(0, \rho) \subseteq R^{N},
$$

for some $y_{0} \neq 0 \in R^{m}$ and $\varphi \in C^{1}\left(D \supseteq B\left(y_{0}, a\right)\right)$, where $B\left(y_{0}, a\right) \subseteq\left\{y\left(p ; y_{0}\right) \neq 0: p \in B(0, \rho) \subseteq R^{N}\right\}$, then $\partial_{y} \varphi(y)=0 \in R^{m}, \forall y \in B\left(y_{0}, a\right)$.

The conclusion (22) relies on Theorem 1 and we get

$$
\begin{gathered}
\operatorname{dim} L\left(Y_{1}, \ldots, Y_{N}\right)\left(y_{0}\right)=\operatorname{dim}\left[\operatorname{span}\left\{Y_{1}\left(y_{0}\right), \ldots, Y_{N}\left(y_{0}\right)\right\}\right] \\
=\operatorname{dim}\left[\operatorname{span}\left\{C_{1} y_{0}, \ldots, C_{N} y_{0}\right\}\right]=\operatorname{dim}\left\{C y_{0}: C \in M_{m \times m}\right\}=m, \quad \forall y_{0} \neq 0 \in R^{m} .
\end{gathered}
$$

Using (23) and (24), we compute the following Lie derivatives

$$
\begin{gathered}
0=<\partial_{p}\left[\varphi\left(y\left(p ; y_{0}\right)\right)\right], A^{-1}(p) e_{j}>=<\partial_{y} \varphi\left(y\left(p ; y_{0}\right)\right), \partial_{p} y\left(p ; y_{0}\right) A^{-1}(p) e_{j}> \\
=<\partial_{y} \varphi\left(y\left(p ; y_{0}\right)\right), Y_{j}\left(y\left(p ; y_{0}\right)\right)>, \quad j \in\{1,2, \ldots, N\},
\end{gathered}
$$

where $\left\{y\left(p ; y_{0}\right) \neq 0: p \in B(0, \rho)\right\} \supseteq B\left(y_{0}, a\right)$ and $\left\{e_{1}, e_{2}, \ldots, e_{N}\right\} \subseteq R^{N}$ is the canonical basis. Taking into account (22) and (26), we obtain $\partial_{y} \varphi\left(y\left(p ; y_{0}\right)\right)=0, p \in B(0, \rho)$, and $\partial_{y} \varphi(y)=0$ for any $y \in B\left(y_{0}, a\right)$. In other words, as far as

$$
\operatorname{dim}\left[\operatorname{span}\left\{Y_{1}\left(y_{0}\right), \ldots, Y_{N}\left(y_{0}\right)\right\}\right]=m,
$$


for any $y_{0} \neq 0$ (see (22)), from (26), we obtain both $\partial_{y} \varphi\left(y\left(p ; y_{0}\right)\right)=0 \in R^{m}$ for any $p \in B(0, \rho) \subseteq R^{N}$ and $\partial_{y} \varphi(y)=0 \in R^{m}$, for any $y \in B\left(y_{0}, a\right) \subseteq R^{m}$.

Theorem 2. Assume $a_{j}(t) \equiv 0, j \in\{1,2, \ldots, i\}$, for some $1 \leq i \leq m$. Define a subspace $S_{i}=$ span $\left\{e_{1}, \ldots, e_{i}\right\} \subseteq R^{m}$ and its orthogonal complement $S_{i}^{\perp} \subseteq R^{m}$, where $\left\{e_{1}, \ldots, e_{m}\right\} \subseteq R^{m}$ is the canonical basis. Then

$\forall y_{0} \in S_{i}$ is a stationary point for ODE (12).

In addition, for each $y_{0} \in S_{i}^{\perp}, y_{0} \neq 0$, the following statements are valid:

$$
\begin{gathered}
\operatorname{dim} L\left(Y_{j(i)}, \ldots, Y_{N}\right)\left(y_{0}\right)=\operatorname{dim}\left[\operatorname{span}\left\{Y_{j(i)}\left(y_{0}\right), \ldots, Y_{N}\left(y_{0}\right)\right\}\right]=m-i, \\
\left\{C_{j(i)}, \ldots, C_{N}\right\} \text { is a basis for } M_{m \times m}^{i}:=L\left(C_{j(i)}, \ldots, C_{N}\right), j(i):=m i+1 \\
\hat{y}\left(t, y_{0}\right) \in S_{i}^{\perp}, t \in I, \text { for any solution of } O D E(12) .
\end{gathered}
$$

Proof. As far as any $y_{0} \in S_{i}$ is a stationary point for each $Y_{j}(y)=C_{j} y, j=j(i), \ldots, N, j(i)=m i+1$, and ODE (12) is written using only $\left\{Y_{j(i)}(y), \ldots, Y_{N}(y)\right\}$, we get the conclusion (27) is satisfied. By definition, (see Theorem 1) the matrices $\left\{C_{j(i)}, \ldots, C_{N}\right\} \subseteq M_{m \times m}^{i}$ determine a basis in the space $M_{m \times m}^{i}$ consisting of all $(m \times m)$ matrices $C=[\underbrace{0 \ldots 0}_{i-\text { times }} c_{i+1} \ldots c_{m}]$, with $c_{j} \in R^{m}, j=i+1, \ldots, m$. Using

$$
\begin{aligned}
\operatorname{dim} L\left(Y_{j(i)}, \ldots, Y_{N}\right)\left(y_{0}\right)=\operatorname{dim}\left[\operatorname{span}\left\{Y_{j(i)}\left(y_{0}\right), \ldots, Y_{N}\left(y_{0}\right)\right\}\right] \\
=\operatorname{dim}\left[\operatorname{span}\left\{C_{j(i)} y_{0}, \ldots, C_{N} y_{0}\right\}\right] \\
=\operatorname{dim}\left\{C^{i} y_{0}: C^{i} \in M_{m \times m}^{i}\right\}=m-i
\end{aligned}
$$

we get the conclusion (28). Notice that $\frac{d}{d t}<\hat{y}\left(t ; y_{0}\right), e_{j}>=0, t \in I$, and $<\hat{y}\left(t ; y_{0}\right), e_{j}>=<y_{0}, e_{j}>=$ $0, t \in I$, for any $j=\overline{1, i}$, which stands for the conclusion (29). The proof is complete.

\section{Conclusions}

In this paper, we investigated (by using the characteristic system method) the kernel of a polynomial of scalar derivations by solving Cauchy Problems for the corresponding system of ODEs. In addition, a gradient representation for the associated Cauchy Problem solution has been formulated. Moreover, as further research directions, some applications were highlighted in this study of some higher-order hyperbolic, parabolic or Hamilton-Jacobi equations involving a finite set of derivations.

Funding: The APC was funded by University Politehnica of Bucharest, "PubArt" program.

Acknowledgments: The author would like to thank the referees for their precise remarks, which improved the presentation of this paper.

Conflicts of Interest: The author declares no conflict of interest.

\section{References}

1. Vârsan, C. Applications of Lie Algebras to Hyperbolic and Stochastic Differential Equations; Kluwer Academic Publishers: Amsterdam, The Netherlands, 1999. 
2. Barbu, V.; Lasiecka, I.; Tiba, D.; Vârsan, C. Analysis and Optimization of Differential Systems. In Proceedings of the IFIP TC7/WG7.2 International Working Conference on Analysis and Optimization of Differential Systems, Constanta, Romania, 10-14 September 2002; Kluwer Academic Publishers: Amsterdam, The Netherlands, 2003.

3. Iftimie, B.; Marinescu, M.; Vârsan, C. Functionals Associated with Gradient Stochastic Flows and Nonlinear SPDEs, Proceedings of AMAMEF Conferences; Springer: Berlin/Heidelberg, Germany, 2011; pp. 397-417.

4. Friedman, A. Stochastic Differential Equations and Applications; Academic Press: New York, NY, USA, 1975; Volume 1.

5. Sussmann, H.J. Lie brackets and local controllability: A sufficient condition for scalar-input systems. SIAM J. Control Optim. 1983, 21, 686-713. [CrossRef]

6. Crandall, M.G.; Souganidis, P.E. Developments in the theory of nonlinear first-order partial differential equations. In North-Holland Mathematics Studies; North-Holland: Amsterdam, The Netherlands, 1984.

7. Sontag, E.D. Mathematical Control Theory: Deterministic Finite Dimensional Systems; Texts Appl. Math. 6; Springer-Verlag: New York, NY, USA, 1990.

8. Bressan, A.; Shen, W. On Discontinuous Differential Equations. In Differential Inclusions and Optimal Control; Andres, J., Gorniewicz, L., Nistri, P., Eds.; J. Schauder Center, Lecture Notes in Nonlinear Analysis; Kluwer Academic Press: New York, NY, USA, 1998; Volume 2, pp. 73-87.

9. Evans, L.C. An Introduction to Mathematical Optimal Control Theory; Lecture Notes; Department of Mathematics, University of California: Berkeley, CA, USA, 2008.

10. Brezis, H. Functional Analysis, Sobolev Spaces and Partial Differential Equations; Springer: Berlin, Germany, 2011.

11. Parveen, S.; Akram, M.S. Linear subspaces of smooth vector fields as a kernel of some linear first order partial differential equations. Syst. Control Lett. 2011, 61, 86-91. [CrossRef]

12. tă, S.T.; Vârsan, C. Weak small controls and approximations associated with controllable affine control systems. J. Differ. Equ. 2013, 255, 1867-1882.

13. tă, S.T. Local uncontrollability for affine control systems with jumps. Int. J. Control 2017, 90, 1893-1902.

(c) 2020 by the author. Licensee MDPI, Basel, Switzerland. This article is an open access article distributed under the terms and conditions of the Creative Commons Attribution (CC BY) license (http:/ / creativecommons.org/licenses/by/4.0/). 